\title{
Breast Cancer Patient Prognosis Is Determined by the Interplay between TP53 Mutation and Alternative Transcript Expression: Insights from TP53 Long Amplicon Digital PCR Assays
}

\author{
Annette Lasham 1,2,*(D), Nicholas Knowlton ${ }^{1,2}$, Sunali Y. Mehta 2,3 ${ }^{\mathbb{D}}$, Antony W. Braithwaite $2,3,4,+$ \\ and Cristin G. Print $1,2,+$ (D) \\ 1 Department of Molecular Medicine and Pathology, School of Medical Sciences, University of Auckland, \\ Auckland 1142, New Zealand; n.knowlton@auckland.ac.nz (N.K.); c.print@auckland.ac.nz (C.G.P.) \\ 2 Maurice Wilkins Centre, University of Auckland, Auckland 1010, New Zealand; \\ sunali.mehta@otago.ac.nz (S.Y.M.); antony.braithwaite@otago.ac.nz (A.W.B.) \\ 3 Department of Pathology, University of Otago, Dunedin 9016, New Zealand \\ 4 Malaghan Institute of Medical Research, Wellington 6242, New Zealand \\ Correspondence: a.lasham@auckland.ac.nz \\ + These authors contributed equally.
}

check for updates

Citation: Lasham, A.; Knowlton, N.; Mehta, S.Y.; Braithwaite, A.W.; Print, C.G. Breast Cancer Patient Prognosis Is Determined by the Interplay between TP53 Mutation and Alternative Transcript Expression: Insights from TP53 Long Amplicon Digital PCR Assays. Cancers 2021, 13, 1531. https://doi.org/10.3390/ cancers13071531

Academic Editors:

Jean-Christophe Bourdon and Neda Slade

Received: 25 February 2021

Accepted: 22 March 2021

Published: 26 March 2021

Publisher's Note: MDPI stays neutral with regard to jurisdictional claims in published maps and institutional affiliations.

Copyright: (c) 2021 by the authors. Licensee MDPI, Basel, Switzerland. This article is an open access article distributed under the terms and conditions of the Creative Commons Attribution (CC BY) license (https:/ / creativecommons.org/licenses/by/ $4.0 /)$.
Simple Summary: The TP53 gene, the most commonly mutated gene in human cancers, is capable of producing multiple RNAs (transcripts). The aim of our study was to measure the abundance of each TP53 transcript, combined with TP53 gene mutation information, to determine the interplay between these in a cohort of breast tumors from New Zealand patients. To do this, we devised a new assay which then enabled the measurement of all known TP53 transcripts. We showed how TP53 gene mutations influenced the levels of specific TP53 transcripts in breast tumors. We evaluated whether a combination of TP53 tumor information, including TP53 mutation status and the levels of certain TP53 transcripts, with standard clinical and pathological information, was associated with breast cancer patient outcome. We recommend that a truly comprehensive analysis of TP53 needs to incorporate data about both TP53 DNA mutations and the expression of the alternative TP53 transcripts.

Abstract: The TP53 gene locus is capable of producing multiple RNA transcripts encoding the different p53 protein isoforms. We recently described multiplex long amplicon droplet digital PCR (ddPCR) assays to quantify seven of eight TP53 reference transcripts in human tumors. Here, we describe a new long amplicon ddPCR assay to quantify expression of the eighth TP53 reference transcript encoding $\Delta 40 \mathrm{p} 53 \alpha$. We then applied these assays, alongside DNA sequencing of the TP53 gene locus, to tumors from a cohort of New Zealand (NZ) breast cancer patients. We found a high prevalence of mutations at TP53 splice sites in the NZ breast cancer cohort. Mutations at TP53 intron 4 splice sites were associated with overexpression of $\triangle 133 T P 53$ transcripts. Cox proportional hazards survival analysis showed that interplay between TP53 mutation status and expression of TP53 transcript variants was significantly associated with patient outcome, over and above standard clinical and pathological information. In particular, patients with no TP53 mutation and a low ratio of TP53 transcripts $t 2$ to $t 1$, which derive from alternative intron 1 acceptor splice sites, had a remarkably good outcome. We suggest that this type of analysis, integrating mutation and transcript expression, provides a step-change in our understanding of TP53 in cancer.

Keywords: TP53 isoforms; alternative splicing; breast cancer prognosis; long amplicon digital PCR

\section{Introduction}

The tumor suppressor protein p53, encoded by the TP53 gene, is the most commonly mutated gene in human cancers [1]. In international breast cancer datasets, somatic TP53 
mutations have been reported to occur in $22.8-34 \%$ of cases [2-4]. TP53 is the most frequently mutated gene in more aggressive breast cancer subtypes, the estrogen receptor (ER) negative Her2+ and triple negative (TNBC) breast cancers, where TP53 mutations occur in more than $50 \%$ of cases [3-6]. A recent integrative analysis of the role of TP53 in over 10,000 patient samples from 32 cancer types, combining TP53 copy number, mutation status and whole TP53 gene locus-level RNA-seq data showed that TP53 RNA abundance was influenced by the presence and type of TP53 mutation [4]. These findings are in accord with our previous demonstration in breast cancer that specific classes of TP53 mutation are associated with enhanced or reduced TP53 locus expression [7]. The TP53 gene locus is capable of expressing multiple RNA transcripts, generated by alternative gene promoter usage and alternative RNA splicing, which encode multiple p53 protein isoforms (Figure 1; [8]).

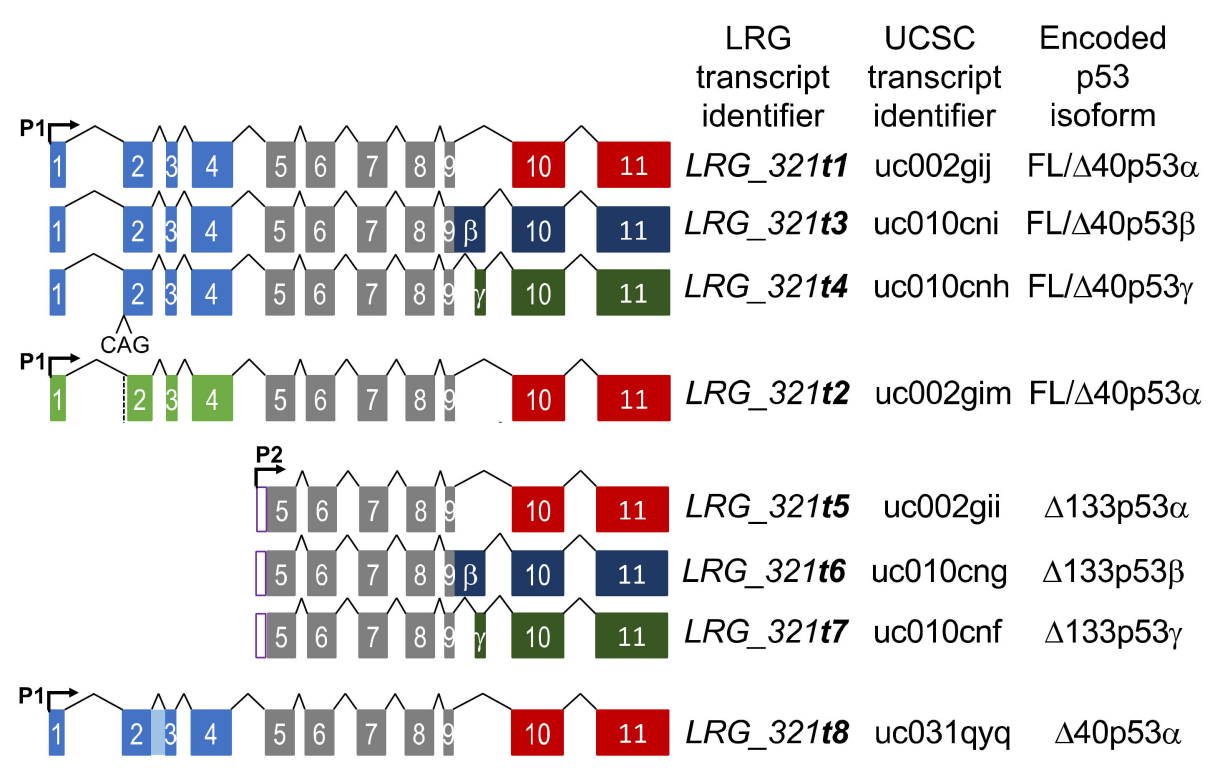

Figure 1. Schematic showing the structure of the eight TP53 reference transcripts. The Locus Reference Genome (LRG) transcript identifier, the UCSC transcript identifier and the predicted encoded p53 isoforms are also shown for each TP53 transcript. P1 and P2 refer to the P1 and P2 promoters, respectively. The light blue and light green boxes refer to transcripts with or without an additional CAG at the beginning of exon 2, respectively. The shaded light blue box between exons 2 and 3 is retention of intron 2 within the $\Delta 40 \mathrm{p} 53$-encoding transcript, $t 8$. The grey boxes indicate the exons common to all transcripts. The red, dark blue and dark green boxes refer to transcripts encoding $\alpha, \beta$ and $\gamma$ C-terminal ends.

The Ensembl locus reference genomic record for TP53 (LRG_321) describes eight reference transcripts, $t 1-t 8[9,10]$, although more are predicted [11]. All TP53 transcripts have a common region of $618 \mathrm{bp}$, spanning exons 5-9. Until recently, the length of this common region prevented the end-to-end detection required for precise quantitation of the individual RNA transcripts by standard RT-qPCR or short-read RNA-sequencing $[12,13]$. Therefore, what is currently known of the range of TP53 transcripts has focused on quantitation of either the $5^{\prime}$ or $3^{\prime}$ end sequences, predominantly using RT-qPCR [11]. This approach has shown that the levels of the isoform "ends" are associated with various clinical or pathological features of breast cancer. For example, elevated levels of $\triangle 40 T P 535^{\prime}$ ends have been observed in breast compared to normal tissues [14]. The different TP53 RNA transcripts play distinct biological roles and in breast cancer, the TP53 $\beta 3^{\prime}$ end levels have been negatively associated with tumor size, and positively associated with estrogen receptor (ER) positive tumors and better patient outcomes [14,15]. Higher expression of the TP53 $3^{\prime}$ end in breast cancers with a mutant p53 has been associated with good patient 
prognosis [15]. Using nested PCR to detect the transcript encoding the $\triangle 133 p 53 \beta$ isoform, breast cancer patients with the highest levels had the poorest prognosis [16].

In the accompanying manuscript, we describe multiplex long amplicon ddPCR assays to quantitate seven of the individual TP53 reference transcripts in cell lines and freshfrozen breast tumors [13]. We showed that five of these TP53 reference transcripts were coexpressed in all breast tumors analyzed and that non-canonical TP53 transcripts could also be identified [13]. Depending on their position, TP53 DNA mutations will be transcribed into some but not all TP53 RNA transcripts, altering the biological function of only that subset of p53 protein isoforms that incorporate the translated mutations. In addition, TP53 DNA mutations may alter splicing patterns and influence the relative abundance of the various alternative TP53 RNAs and their translated protein isoforms. Therefore, a truly comprehensive analysis of TP53 needs to incorporate data about both TP53 DNA mutations and the expression of the alternative TP53 RNA transcripts.

In this study, we combine gene sequencing with long amplicon ddPCR assays to integrate information about TP53 transcript abundance, TP53 mutation status and clinical and pathological information in cohort of tumors from 89 breast cancer patients. Our analysis includes a new multiplex long amplicon ddPCR assay to detect the final TP53 reference transcript, $L R G \_321 t 8$, encoding $\triangle 40 \mathrm{p} 53 \alpha$, thereby providing information about all eight TP53 reference transcripts. We observe a high number of TP53 splice site mutations in this breast cancer cohort, which we find influence TP53 transcript variant expression. We also show that information about TP53 mutations and the abundance of alternatively spliced TP53 RNA transcripts is associated with patient outcome and strengthens prognostic associations when added to standard clinical and pathological information.

\section{Results}

\subsection{A Cohort of New Zealand Breast Cancers Has a High Proportion of TP53 Splicing Mutations}

We mapped somatic TP53 mutations in breast tumors of 89 New Zealand patients using targeted sequencing of the TP53 gene locus. This identified 31 tumors with mutations that influence the TP53 coding region, accounting for $\sim 35 \%$ of the cohort (Table S1). These mutations occurred along the length of the TP53 gene, with $45 \%$ being missense (Table 1 and Figure 2).

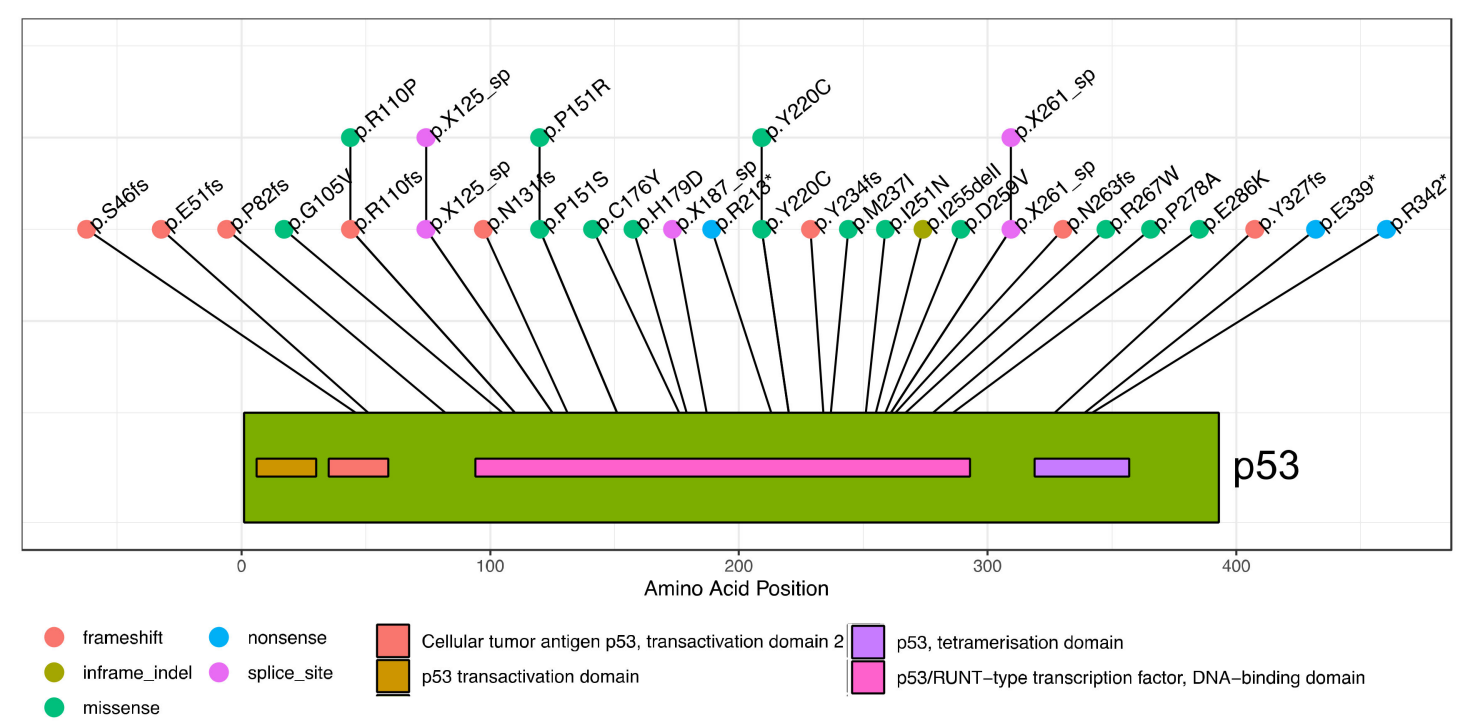

Figure 2. Lollipop plot showing location and sequence of TP53 mutations in breast cancer cohort. The individual mutations are indicated with their amino acid change given. Mutations preceded with " $\mathrm{X}$ " are splice site mutations, located within introns. The color of the lollipop indicates the mutation type. A schematic of the p53 protein is shown, with domains indicated in the color key below the plot. 
Table 1. Description and frequency of TP53 mutations in New Zealand breast cancer cohort.

\begin{tabular}{ccc}
\hline TP53 Mutation Type & Frequency & Number of Tumors \\
\hline Frameshift & $26 \%$ & 8 \\
Missense & $45 \%$ & 14 \\
Nonsense & $10 \%$ & 3 \\
Splicing & $16 \%$ & 5 \\
In-Frame indel & $3 \%$ & 1 \\
\hline
\end{tabular}

While we did not observe some TP53 hotspot mutations commonly reported in international breast cancer studies such as: R175, R248, R273, we did observe many frequent mutations including R110, H179, R213*, Y220C, R342* [3,6,17-19].

\subsection{Detailed Analysis of TP53 Splicing Mutations and Their Consequences on RNA Transcript Expression}

We noted a moderate proportion of mutations predicted to influence TP53 transcript splicing (16\%). These include mutations within intron 4, intron 5 and intron 7 (Table S1). To confirm and quantitate the TP53 transcripts expressed in tumors with these mutations, we performed long amplicon ddPCR assays between specific exons using cDNA prepared from RNA as template to confirm that all TP53 splice mutations were expressed. For tumors with intron 4 splice site mutations, AL0021 and AL0060, these both had mutations within the donor splice site, at +1 and +5 , respectively. Although an additional transcript retaining the 757 bp intron 4 was clearly evident in the $t 1$ long amplicon ddPCR assays, there was insufficient separation between the two amplified products to accurately quantitate both transcripts [13]. Therefore, we designed specific ddPCR assays to amplify a region of TP53 RNA between exons 3 and 6, which generated a smaller amplicon able to clearly resolve and quantitate TP53 RNAs retaining intron 4. This confirmed that both the AL0021 and AL0060 tumors expressed two TP53 transcripts, with and without retention of intron 4 (amplicon sizes 1,293 bp and 536 bp, respectively, Figure 3a). cDNA from tumor AL0001 was used as a control to show amplification of canonically spliced TP53 RNA, and genomic DNA to demonstrate the amplicon size when TP53 introns 3, 4 and 5 are retained (amplicon size of 1,483 bp). From the TP53 gene sequencing, the mutant allele frequency was $58 \%$ and $53 \%$ (Table S1), and quantitation of the products of these ddPCR assays showed that the alternative (intron 4-retaining) transcript comprised $49 \%$ and $40 \%$ of the TP53 transcripts for tumors AL0021 and AL0060, respectively.

Tumors AL0034 and AL0073 had mutations that we predicted would affect the correct splicing of intron 7. AL0034 had a 21 bp deletion that spanned 14 bases at the $3^{\prime}$ end of exon 7 and the first 7 bases in intron 7, and AL0073 had a point mutation at +1 in the intron donor splice site. To test this prediction, a ddPCR assay was designed to amplify TP53 RNA between exons 7 and 8 to determine whether intron 7 was retained in some TP53 transcripts. This confirmed that both AL0034 and AL0073 had TP53 RNAs that retained intron 7 (amplicon size 463/484 bp, respectively), in addition to a canonically spliced RNA (amplicon size 141 bp; Figure 3b). cDNA from tumor AL0001 was used as a control to show amplification of spliced TP53 RNA and genomic DNA to show when intron 7 was retained. From the TP53 gene sequencing we had observed that the mutant allele frequency was $32 \%$ and 39\% for AL0034 and AL0073, respectively. From quantitation of the individual ddPCR products in this assay, the alternative (intron 7-retaining) RNAs comprised $36 \%$ and $82 \%$ of the TP53 RNAs for AL0034 and AL0073, respectively (Figure 3b). 

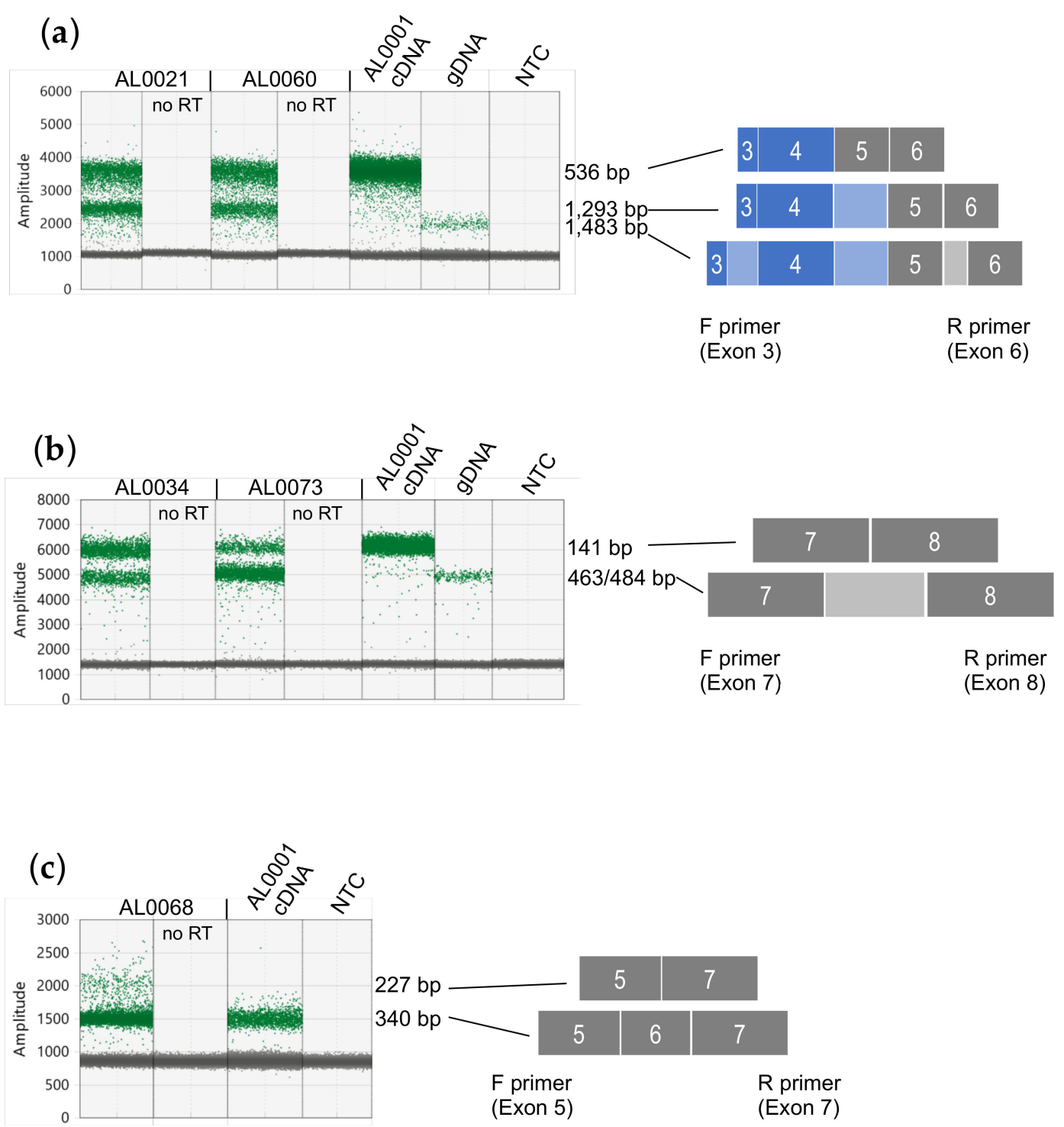

Figure 3. Validation of TP53 splicing mutations in breast cancer samples. (a) ddPCR assay between TP53 exons 3 and 6 showing amplification of TP53 transcripts. AL0021 and AL0060 have a point mutation in the TP53 intron 4 donor splice site at +1 and +5 , respectively. AL0001 has no splicing mutations and was used as a control to show the fluorescence amplitude of a correctly spliced TP53 RNA (536 bp), whereas those TP53 RNAs retaining intron 4 have an amplicon size of $1293 \mathrm{bp}$. Genomic DNA (gDNA) was used as a control template to show an amplicon with retention of introns 3, 4 and 5. (b) ddPCR assay between TP53 exons 7 and 8 showing amplification of TP53 transcripts. AL0034 and AL0073 have a 21 bp deletion spanning the TP53 intron 7 donor splice site or a point mutation at +1 in the TP53 intron 7 donor splice site, respectively. AL0001 has no splicing mutations and was used as a control to show the fluorescence amplitude of a correctly spliced TP53 RNA (141 bp), whereas those TP53 RNAs retaining intron 7 have an amplicon size of 463 or 484 bp for AL0034 and AL0073, respectively. Genomic DNA (gDNA) was used as a control template to show an amplicon with retention of intron 7 (484 bp). (c) ddPCR assay between TP53 exons 5 and 7 showing amplification of TP53 transcripts. AL0068 has a point mutation at -1 in the TP53 intron 5 acceptor splice site. AL0001 has no splicing mutations and was used as a control to show the fluorescence amplitude of a correctly spliced TP53 RNA (340 bp), whereas those TP53 RNAs skipping exon 6 have an amplicon size of $227 \mathrm{bp}$. ddPCR results shown are "1-D" plots, with green dots representing droplets where PCR products have been amplified and grey dots represent no amplified PCR product. The fluorescence amplitude (Amplitude) on the $y$-axes is indicative of amplicon size/s within each assay.

Tumor AL0068 had a low allele frequency splicing mutation (13\%) at -1 of the acceptor splice site of intron 5. This would also be present in all TP53 reference transcripts. A ddPCR assay designed between exons 5 and 7 showed that a TP53 RNA smaller than the expected 
size was also amplified, consistent with this mutation causing omission of exon 6, but not retention of intron 5 (Figure 3c). Quantitation of the individual ddPCR products in this assay showed that RNAs skipping TP53 exon 6 comprised 5\% of the total TP53 transcripts in tumor AL0068. Tumor AL0001 was used as a control to show amplification of spliced TP53 RNA (amplicon size of $340 \mathrm{bp}$ ).

\subsection{TP53 Intron 4 Splice Mutations Are a Mechanism to Overexpress $\triangle 133 T P 53$ Transcripts}

Using our long amplicon ddPCR assays to quantitate the individual TP53 transcripts [13], we observed that both tumors with the intron 4 splicing mutation (AL0021 and AL0060) expressed extremely high levels of the $t 5$ transcript, encoding the $\Delta 133$ p $53 \alpha$ isoform, compared to samples in the rest of the cohort (Figure 4a).

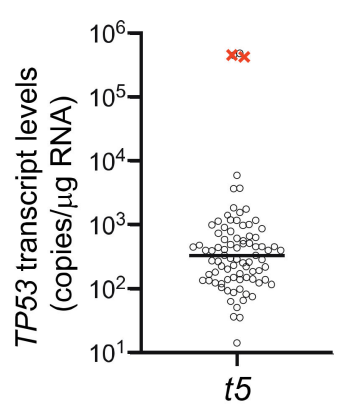

(a)

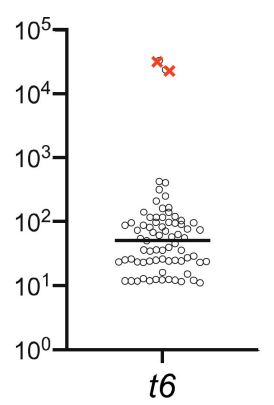

(b)

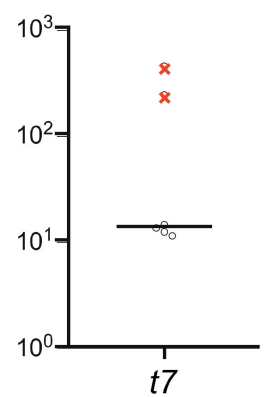

(c)

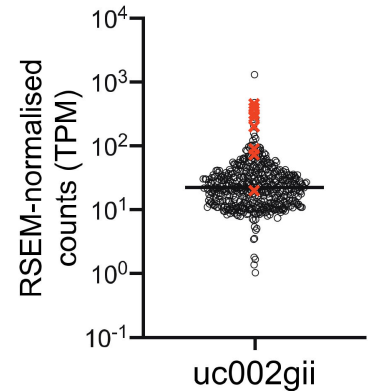

(d)

Figure 4. Scatterplots showing levels of transcripts encoding $\Delta 133$ p53 isoforms in Breast Cancer cohorts. (a-c) Results from long amplicon ddPCR of 85 NZ breast cancer samples with both known TP53 mutation status and TP53 transcript expression data. (a) $L R G \_321 t 5$ encoding $\Delta 133 p 53 \alpha$ (detectable in 83/85 tumors), (b) LRG_321t6 encoding $\Delta 133 p 53 \beta$ (detectable in 73/85 tumors), (c) $L R G \_321 t 7$ encoding $\triangle 133$ p53 $\gamma$ (detectable in 6/85 tumors) and (d) uc002gii TP53 transcript, assigned as encoding $\Delta 133$ p $53 \alpha$, quantitated by RSEM analysis of RNA-seq data in 727 TCGA BRCA samples with known TP53 mutation status (TPM = transcripts per million reads). Black circles represent tumor samples expressing this transcript; for clarity samples have been jittered on the $x$-axis. Tumors with a TP53 intron 4 splice site mutation are indicated by $\times$. Horizontal line represents the median expression level for each cohort. Note that "detectable" refers to limit of detection of ddPCR assays, which is transcripts with abundance greater than 10 copies/ $\mu$ g RNA.

These tumors also expressed extremely high levels of the $t 6$ and $t 7$ transcripts (Figure $4 \mathrm{~b}, \mathrm{c}$; for transcript positions refer to Figure 1). In order to determine whether this was a feature of TP53 intron 4 splice mutations, we examined RNA-seq data from the TCGA BRCA cohort $[20,21]$. 727 samples that had both TP53 mutation status reported and expressed the transcript assigned to encode $\Delta 133 \mathrm{p} 53 \alpha$ (transcript uc002gii, referred to as $t 5$ here; see Figure 1), were analyzed. Eight BRCA tumors were reported to have a TP53 intron 4 splice site mutation, and consistent with our results, seven of these expressed the uc002gii transcript at $\geq 95$ th percentile (Figure $4 \mathrm{~d}$ ). Transcripts assigned as encoding $\Delta 133 \mathrm{p} 53 \beta$ (uc010cng, referred to as $t 6$ here) and $\Delta 133 \mathrm{p} 53 \gamma$ (uc010 cnf, referred to as $t 7$ here) were only measurable by RNA-seq in $10 \%$ and $1.8 \%$ of samples, respectively, so were not included in this analysis. Since intron 4 contains the $5^{\prime}$ untranslated region of the $\triangle 133 T P 53$ transcripts, these transcripts are not $t 5-t 7$ per se (i.e., driven off the P2 promoter), but are $t 1, t 4$ and $t 5$ transcripts retaining intron 4 , allowing the P1 promoter to drive expression of transcripts that have the $t 5-t 7$-specific $5^{\prime}$ end sequences. This suggests that TP53 intron 4 splice mutations may provide a mechanism to overexpress transcripts encoding the $\Delta 133$ p53 isoforms.

\subsection{Analysis of All TP53 Reference Transcripts in New Zealand Breast Cancer Cohort}

Our previous work described the long amplicon ddPCR assays that were used to quantitate the expression of seven TP53 transcripts encoding FL $/ \triangle 40 \mathrm{p} 53 \alpha, \beta$ and $\gamma$, and $\Delta 133$ p $53 \alpha, \beta$ and $\gamma$ (LRG_321 transcripts $11-t 7$; see Figure 1 ) in a New Zealand breast cancer 
cohort [13]. Building on this work and using an additional ddPCR assay to quantitate TP53 reference transcript $L R G \_321 t 8$ that encodes the $\Delta 40 \mathrm{p} 53 \alpha$ isoform, we aimed to determine whether the relative abundance of the eight individual TP53 reference transcripts and the mutations they carry were associated with any clinical or pathological features.

\subsubsection{Quantitation of $\Delta 40 \mathrm{p} 53$-Encoding Transcripts}

The new assay to quantitate reference transcript $L R G \_321 t 8$ differed from the previous designs by the forward primer being located with intron 2 [22]. This allowed detection and quantitation of the $t 8$ transcript, encoding $\Delta 40 \mathrm{p} 53 \alpha$, and also any $\beta$ or $\gamma$-encoding $\Delta 40 \mathrm{p} 53$ transcripts (Figure S1a). In the New Zealand breast cancer cohort we found that the $t 8$ transcript was detectable in all tumors (from 67 to 17,169 copies/ $\mu$ g RNA), with a $\Delta 40 \mathrm{p} 53 \beta$ encoding transcript detectable in 51 tumors (referred to as $t 8 \beta$; from 32 to 714 copies $/ \mu \mathrm{g}$ RNA) and a $\Delta 40$ p53 $\gamma$-encoding transcript detectable in only 2 tumors (referred to as $t 8 \gamma$; 33 and 35 copies/ $\mu$ g RNA) (Figure S1b). Plotting the abundance of all TP53 transcripts in this cohort showed that the $t 8$ transcript was of similar abundance to the TP53 $t 2$ and $t 3$ transcripts (encoding FL/ $\Delta 40 \mathrm{p} 53 \alpha$ and FL/ $\Delta 40 \mathrm{p} 53 \beta$, respectively; Figure S1b).

\subsubsection{Association of TP53 Mutation Type with Expression of Individual TP53 RNA Transcripts}

We next examined the relationship between TP53 mutation type and expression levels of individual TP53 RNA transcripts. (Figure 5a-i). In our cohort, 85/89 breast tumors had both TP53 transcript and TP53 mutation information. Although the numbers were small, analysis of abundance of transcripts encoding FL or $\Delta 40 \mathrm{p} 53$ isoforms with an $\alpha \mathrm{C}$-terminal end (transcripts $t 1, t 2, t 8$ ) showed that those with a frameshift mutation were present at lower levels than those $\alpha$-encoding transcripts with a missense or no TP53 mutation (e.g., for $t 1$ transcripts encoding FL/ $\triangle 40 T P 53 \alpha, p=0.017$, Figure 5a), concordant with our previous finding analyzing the whole TP53 gene locus-level expression [7]. However, we did not observe this for transcripts encoding FLp53 or $\Delta 40$ p53 isoforms with a $\beta$ Cterminal end (transcripts $t 3$ and $t 8 \beta$ ), where there was no association between mutation type and RNA abundance (for $t 3$ transcripts encoding FL/ $\Delta 40 p 53 \beta, p=$ ns, Figure $5 c$ ). We also saw no association between mutation type and RNA abundance for the $t 5$ and t6 transcripts (encoding $\Delta 133 p 53 \alpha$ and $\beta$, respectively), however only four and three frameshift mutations would be present in the $\Delta 133 \mathrm{p} 53 \alpha$ and $\beta$ sequences of these tumors, respectively (Figure 5e,f). To determine whether this observation with transcripts encoding the FL/ $\triangle 40$ p53 $\alpha$ and $\beta$ isoforms was also seen in another cohort, we analyzed RNA-seq data from the TCGA BRCA cohort, by plotting transcript levels of uc002gij.2 and uc010cni.1 (assigned as encoding FL/ $\triangle 40 \mathrm{p} 53 \alpha$ and FL/ $\triangle 40 \mathrm{p} 53 \beta$, respectively) by TP53 mutation type. These results were consistent with the findings from our cohort, that the transcripts encoding the FL/ $\triangle 40$ p53 $\alpha$ protein with TP53 frameshift mutations were less abundant than those with missense or no TP53 mutations $(p<0.0001$, Figure $5 \mathrm{j})$. However, the abundance of transcripts encoding FL/ $\triangle 40 \mathrm{p} 53 \beta$ protein were not significantly different by TP53 mutation type or compared to those with no TP53 mutation ( $p=$ ns, Figure 5k).

We also noted that the expression of the $t 4$ transcript (encoding FL/ $\Delta 40 \mathrm{p} 53 \gamma$ ) was associated with TP53 mutation status. Although only 22 of 85 tumors had detectable levels of the $t 4$ transcript, 12 of these were tumors with TP53 mutations $(p=0.048)$. Interestingly, all five tumors with a TP53 splicing mutation had detectable levels of the $t 4$ transcript (Figure 5d). 
(a)

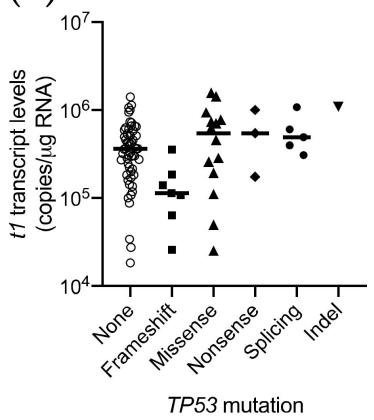

(d)

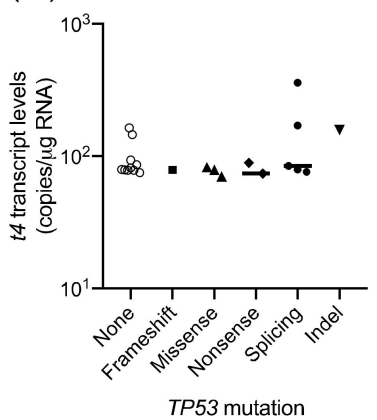

(g)

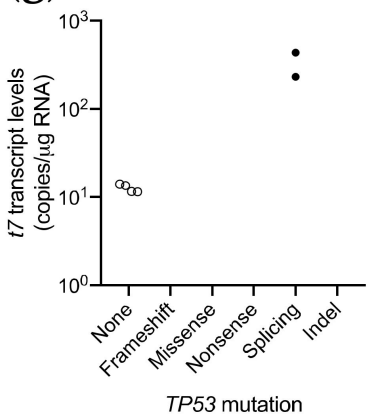

(j)

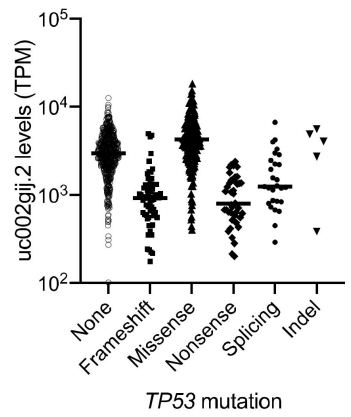

(b)

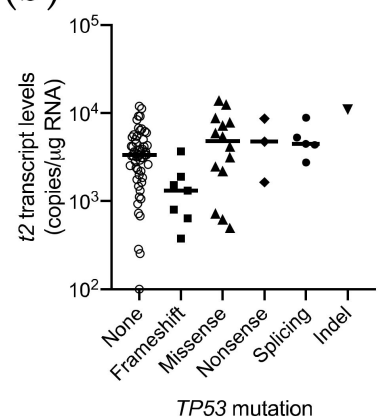

(e)

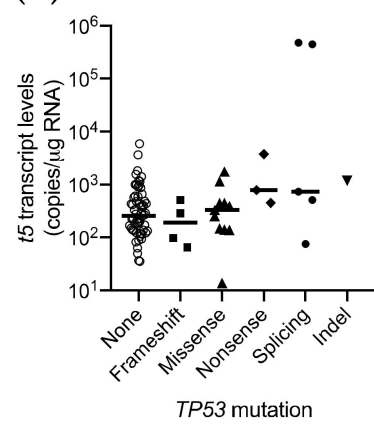

(h)

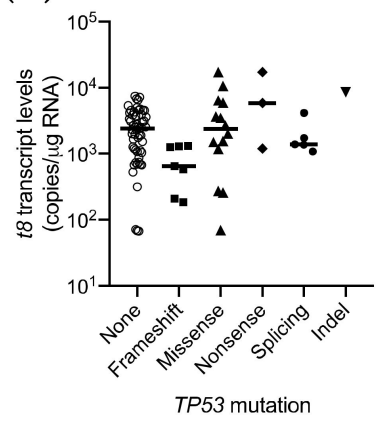

(k)

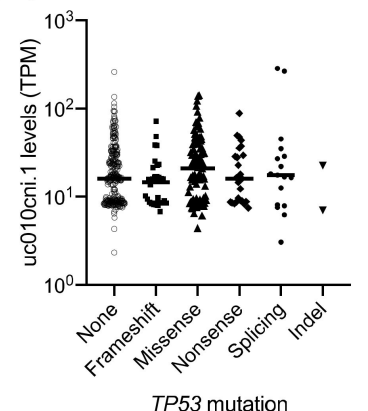

(c)

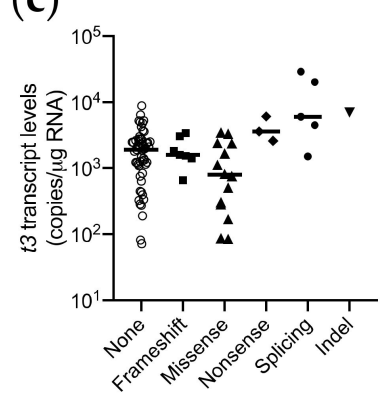

TP53 mutation

(f)

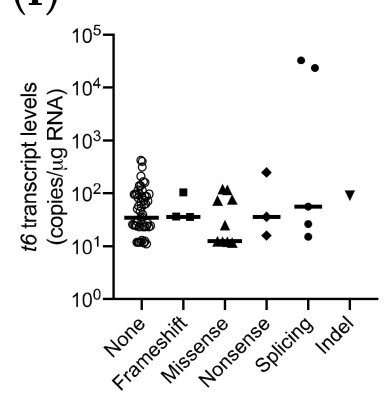

TP53 mutation

(i)

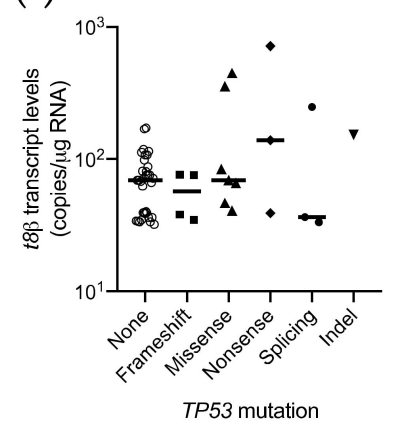

Figure 5. Scatterplots showing TP53 transcript abundance by TP53 mutation status in breast cancer cohorts. (a-i) TP53 transcript levels in New Zealand breast cancer cohort, quantitated by long amplicon ddPCR. (a) $L R G \_321 t 1$ encoding FL/ $\Delta 40$ p53 $\alpha$, (b) $L R G \_321 t 2$ encoding FL/ $\Delta 40 p 53 \alpha$, (c) $L R G \_321 t 3$ encoding FL/ $\Delta 40 \mathrm{p} 53 \beta$, (d) $L R G \_321 t 4$ encoding FL/ $\Delta 40 \mathrm{p} 53 \gamma$, (e) $L R G \_321 t 5$ encoding $\Delta 133 p 53 \alpha$, (f) $L R G \_321 t 6$ encoding $\Delta 133 p 53 \beta$, (g) $L R G \_321 t 7$ encoding $\Delta 133 p 53 \gamma$, (h) $L R G \_321 t 8$ encoding $\Delta 40 \mathrm{p} 53 \alpha$, and (i) $t 8 \beta$ encoding $\Delta 40 \mathrm{p} 53 \beta$, in each tumor. (j,, $\mathbf{k})$ TP53 transcript levels from the TCGA BRCA cohort, quantitated by RNA-seq, (j) uc002gij.2, encoding FL/ $\Delta 40$ p53 $\alpha$, (k) uc010cni.1, encoding FL $/ \triangle 40 \mathrm{p} 53 \beta$. Bar represents the median, with symbols representing individual tumor samples. 


\subsection{TP53 Information Is Associated with Clinical and Pathological Features}

We then determined whether the levels of the transcripts encoding the p53 isoforms were associated with intrinsic breast cancer subtype [23]. Using molecular subtype information generated for these tumors [24], the levels of the individual transcripts were plotted by individual subtype. This showed that in our cohort, the levels of each TP53 transcript were similar across all subtypes (Figure S2; Table S2). Only $t 8$ showed a modest association with subtype $(p=0.042)$, but was not significant after false discovery rate control. These results suggest that the expression of the individual TP53 transcripts is intrinsic to all breast cancers and is not related to breast cancer subtype.

Next we determined whether the levels of the transcripts encoding the p53 isoforms showed any nominal univariate associations with any clinical or pathological features of breast cancer. We used two approaches; either using the concentration of the individual TP53 transcripts (i.e., copies of each transcript/ $\mu$ g tumor RNA) or the ratio of each of the individual TP53 transcripts to the $t 1$ transcript (encoding FL/ $\Delta 40 \mathrm{p} 53 \alpha$ ), as this was the most abundant transcript, and since the use of relative transcript levels provides an internal control for sample-specific factors such as differences in efficiency of cDNA synthesis between tumor samples. We also quantitated the TP53 $5^{\prime}$ and $3^{\prime}$ ends as previously [12] and included these in the analysis. The most significant univariate associations were the t3/t1 ratio with tumor ER status $\left(p=4.7 \times 10^{-4}\right)$, PgR status $\left(p=1.2 \times 10^{-3}\right)$ and breast cancer subtype $\left(p=9.8 \times 10^{-5}\right)$ (Table S2).

We then determined whether other TP53 information, such as TP53 gene mutation status or likely loss of the wild type TP53 allele ( $\mathrm{LOH}$; loss of heterozygosity), was associated with any clinical or pathological features. This showed that the TP53 mutation status was strongly associated with breast cancer subtype $\left(p=4.7 \times 10^{-5}\right)$, tumor ER $\left(p=1.3 \times 10^{-4}\right)$ and PgR status $\left(p=6.5 \times 10^{-6}\right)$ and histological grade $\left(p=4.4 \times 10^{-6}\right)$ (Table S2). However, TP53 LOH was not associated with any clinicopathological features in this cohort.

\subsection{TP53 Mutation Status and t2/t1 Transcript Ratio Are Associated with Breast Cancer Patient Outcome}

We then evaluated whether the levels of the individual TP53 transcripts and/or the TP53 mutational status were associated with patient prognosis. We observed that TP53 mutational status was associated with prognosis, as patients having tumors with a TP53 mutation were more likely to have a distant metastatic event than those with no TP53 mutation (Figure $6 \mathrm{a}, p=1.5 \times 10^{-3}$ ).

Next we used Cox proportional hazard regression models to determine whether there was any relationship between the various types of TP53 information and patient outcome. For the individual TP53 transcripts, we applied two approaches to evaluate how these were associated with prognosis; either using the concentration of the individual transcripts (i.e., copies of each transcript/ $\mu$ g tumor RNA) or the ratio of each of the individual TP53 transcripts to the $t 1$ transcript (encoding FL/ $\Delta 40 \mathrm{p} 53 \alpha$ ), as this was the most abundant transcript. As univariate tumor markers of distant metastases free survival (DMFS), we observed that the TP53 mutational status, the abundance of TP53 $\alpha 3^{\prime}$ RNA end, the abundance of TP53 transcript $t 2$ to TP53 reference transcript $t 1$, and the ratio of the $\triangle 133 T P 535^{\prime}$ end to the FL/ $\triangle 40 T P 53 \_T 15^{\prime}$ end, were significantly associated with patient prognosis. However, after correcting for the false discovery rate, only the TP53 mutation status and the abundance of TP53 transcript $t 2$ to reference transcript $t 1$ had significant prognostic associations (hazard ratio (HR), 3.19; 95\% confidence intervals (CI), 1.49-6.84; $p=2.9 \times 10^{-3}$ for TP53 mutation status and HR, $1.88 ; 95 \%$ CI, $1.38-2.56 ; p=7.3 \times 10^{-5}$ for TP53 $t 2 / t 1$ ratio; Table 2). Then, the association of these two TP53 tumor markers was analyzed further, by adjusting for patient lymph node status, tumor size, histological grade, and estrogen receptor (ER) and progesterone receptor (PgR) status. We found that the TP53 mutation status or the TP53 t2/t1 ratio provided additional information independent of the clinicopathological features and were both significantly associated with DMFS in all patients (HR, 4.36; 95\% CI, 1.47-12.97; $p=8.1 \times 10^{-3}$ for the TP53 mutation status; HR, $1.85 ; 95 \% \mathrm{CI}, 1.32-2.59 ; p=3.4 \times 10^{-4}$ for $T P 53 t 2 / t 1$ ratio; Table 2$)$. 
(a)

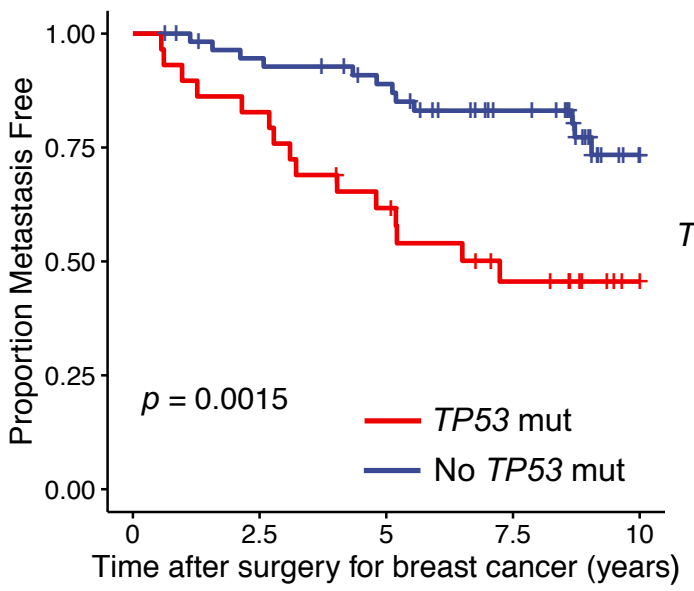

(b)

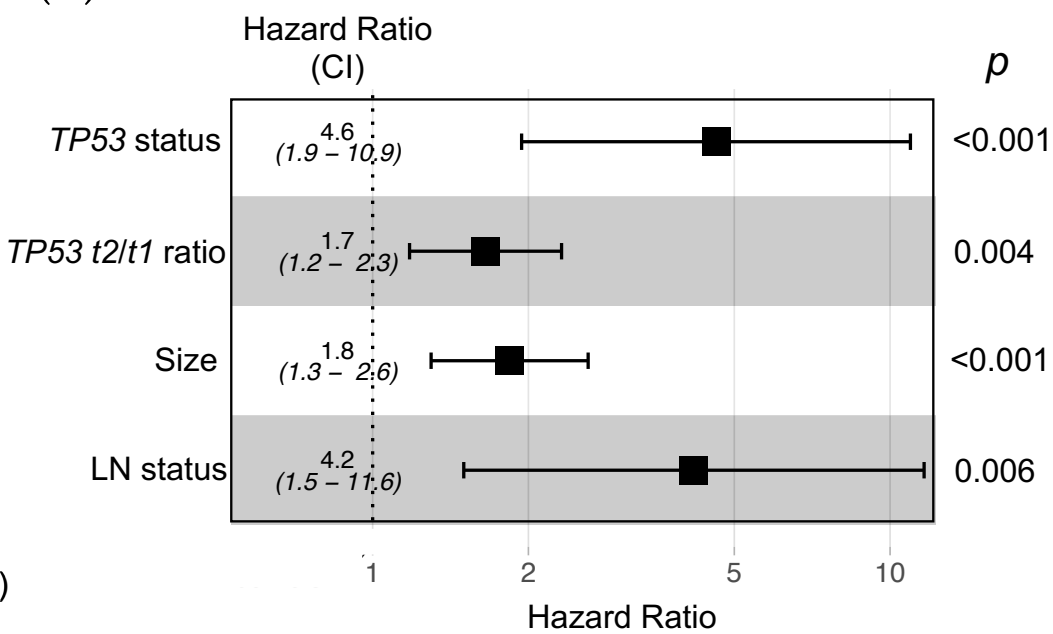

(c)

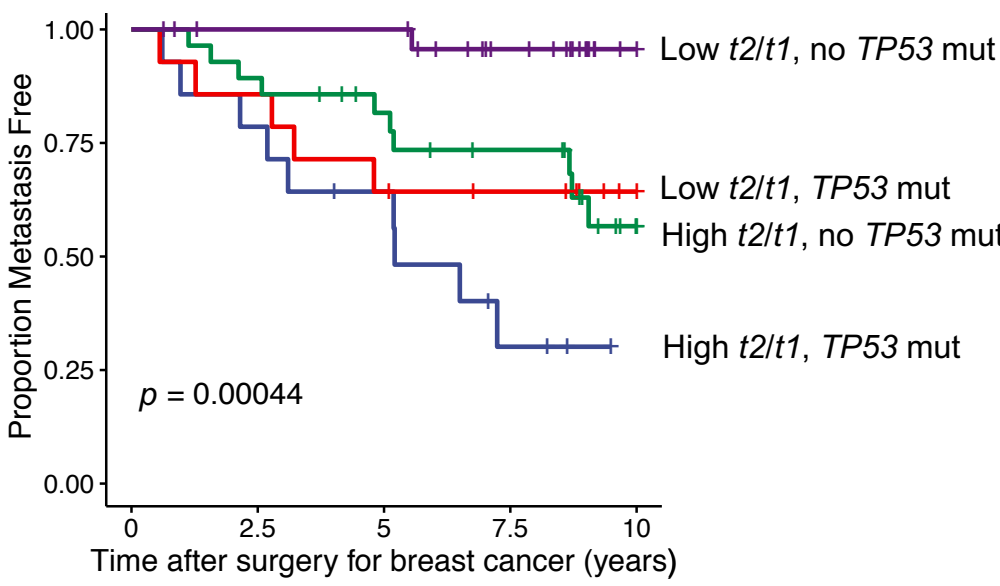

Figure 6. Association of TP53 tumor information with distant metastases free survival in NZ breast cancer patients. (a) Kaplan-Meier curves showing the proportion of patients having a distant metastatic event by their TP53 tumor mutation status ( $\log$ rank test $p=0.0015)$. Red line = patients with tumors with a TP53 mutation $(n=31)$, Blue line = patients with tumors with no TP53 mutation $(n=58)$. (b) Forest plot showing contribution of TP53 tumor information and clinicopathological features to a multivariable Cox proportional hazards model predicting those patients having a distant metastatic event ( $n=83$ with 27 patients developing distant metastases before 12 years, $\log$ rank test $p=4.2 \times 10^{-8}$ ), $\mathrm{CI}=95 \%$ confidence intervals, (c) Kaplan-Meier curves showing the proportion of patients having a distant metastatic event by their TP53 tumor mutation status and $t 2 / t 1$ transcript level ratios to stratify patients into four groups (log rank test $\left.p=4.4 \times 10^{-4}\right)$. "High" = greater than median and "Low" = less than median levels of $t 2 / t 1$, Green line = patients with tumors with High $t 2 / t 1$ levels and no TP53 mutation $(n=27)$, Blue line = patients with tumors with High $t 2 / t 1$ levels and a TP53 mutation $(n=16)$, Purple line = patients with tumors with Low $t 2 / t 1$ levels and no TP53 mutation $(n=28)$ and Red line $=$ patients with tumors with Low $t 2 / t 1$ levels and a TP53 mutation $(n=14)$.

Table 2. Cox univariable analysis of TP53 tumor information showing association with distant metastases free survival (DMFS) of patients with breast cancer.

\begin{tabular}{ccccccc}
\hline $\begin{array}{c}\text { TP53 } \\
\text { Information }\end{array}$ & Raw $\boldsymbol{p}$ Value & $\begin{array}{c}\text { Adjusted } \boldsymbol{p} \\
\text { Value }^{\mathbf{1}}\end{array}$ & Hazard Ratio $^{2}$ & $\begin{array}{c}\text { 95\% Confidence } \\
\text { Intervals }^{2}\end{array}$ & $\begin{array}{c}\text { Raw } \boldsymbol{p} \\
\text { Value }^{\mathbf{2}}\end{array}$ & $\begin{array}{c}\text { Adjusted } \boldsymbol{p} \\
\text { Value }^{\mathbf{1 , 2}}\end{array}$ \\
\hline TP53 mutant & $2.9 \times 10^{-3}$ & 0.037 & 4.36 & $1.47-12.97$ & $8.1 \times 10^{-3}$ & $9.2 \times 10^{-3}$ \\
$t 2 / t 1$ ratio & $7.3 \times 10^{-5}$ & $1.8 \times 10^{-3}$ & 1.85 & $1.32-2.59$ & $3.4 \times 10^{-4}$ & $5.1 \times 10^{-4}$ \\
\hline
\end{tabular}

${ }^{1}$ False discovery rate (fdr)-adjusted $p$ values. ${ }^{2}$ Adjusted for patient lymph node status, tumor size, estrogen receptor and progesterone receptor status and histological grade. 
As a number of risk prediction models for breast cancer patient prognosis have been developed from standard clinico-pathological markers [25-27], we wished to determine whether incorporation of this TP53 information generated an improved prognostic model. Therefore, we performed multivariable analysis, combining clinical and pathological information (tumor ER and PgR status, size, and histological grade, and patient lymph node status) with TP53 mutation status and TP53 t2 transcript abundance relative to the most abundant $t 1$ transcript. These results showed that, with the exception of lymph node status, and tumor size, the combined TP53 genomic information contributed significantly in a Cox proportional hazards model to associate with patients' DMFS, than the other clinicopathological markers (HR, 1.84; 95\% CI, 1.30-2.61; $p=6.3 \times 10^{-4}$ for tumor size; HR, 4.18 ; $95 \%$ CI, 1.50-11.64; $p=6.2 \times 10^{-3}$ for lymph node status; HR, 4.61; 95\% CI, 1.94-10.95; $p=5.4 \times 10^{-4}$ for TP53 mutation status; HR, 1.65; 95\% CI, 1.18-2.32; $p=3.6 \times 10^{-3}$ for TP53 t2/t1 ratio; Table 3.) These results are shown graphically in Figure $6 \mathrm{~b}$.

Table 3. Cox multivariable analysis of TP53 tumor information showing association with DMFS of patients with breast cancer.

\begin{tabular}{cccc}
\hline TP53 Information & Hazard Ratio & 95\% Confidence Intervals & $p$ Value \\
\hline TP53 mutation & 4.61 & $1.94-10.95$ & $5.4 \times 10^{-4}$ \\
$t 2 / t 1$ ratio & 1.65 & $1.18-2.32$ & $3.6 \times 10^{-3}$ \\
Tumor size & 1.84 & $1.30-2.61$ & $6.3 \times 10^{-4}$ \\
Lymph node status & 4.18 & $1.50-11.64$ & $6.2 \times 10^{-3}$ \\
\hline
\end{tabular}

Multivariable Cox models are difficult to visualise, so we plotted a rough approximation using data split into binary categories in Kaplan-Meier plots. Specifically, the patients were stratified into four groups based on the TP53 $t 2 / t 1$ ratio and also the TP53 mutation status of their tumors. This showed that patients with a TP53 tumor mutation, irrespective of the $t 2 / t 1$ ratio, or those with tumors with greater than the median $t 2 / t 1$ ratio with no TP53 mutation had the poorest prognosis (Figure 6c). However, patients with tumors expressing less than the median level of $t 2 / t 1$ ratio and with no TP53 mutation, had very good prognosis, and only one patient in this group developed metastatic disease (Figure 6c). The results from both Figure $6 \mathrm{a}, \mathrm{c}$ demonstrate that even patients with no tumor TP53 mutation can have poor prognosis, but incorporation of TP53 transcript information can be used to further stratify these to identify a group of patients with very good outcomes.

As the levels of the TP53 $\beta$ and TP53 $\gamma 3^{\prime}$ ends in breast tumors have been associated with patient prognosis $[14,15]$, based on TP53 mutation status, we also analyzed how the individual transcripts that would contribute the most to these TP53 $3^{\prime}$ end measurements, that being transcripts $t 3$ (encoding FL/ $\Delta 40 \mathrm{p} 53 \beta$ ) and $t 4$ (encoding FL/ $\Delta 40 \mathrm{p} 53 \gamma$ ), respectively, were associated with distant metastasis-free survival (DMFS) of patients. Patients were stratified by their tumors having a TP53 mutation or not, and then by the level of $t 3$ or $t 4$ transcripts. The Kaplan-Meier plots showed that patients whose tumors had a TP53 mutation and less than median levels of the $t 3$ transcript, or those whose tumors had a TP53 mutation and the $t 4$ transcript was undetectable, were more likely to have poorer prognosis than patients in the other groups (Figure S3). Patients with tumors expressing low t3 transcript levels and with a TP53 mutation had particularly poor prognosis (Figure S3a,c,e). In a small group of patients $(n=10)$ with no TP53 tumor mutation but detectable levels of the $t 4$ transcript, none developed metastatic disease or died from breast cancer before 10 years (Figure S3d,f). However, as these effects had not been seen in a multivariable Cox model with interaction terms (above), this suggested that the associations observed are non-linear or apply to a subset of patients only.

\section{Discussion}

Here, we performed an integrated analysis of TP53 mutation status and expression of the individual TP53 transcripts in tumor samples donated by a cohort of New Zealand breast cancer patients. We also describe a novel long amplicon ddPCR assay to detect the 
final TP53 reference transcript, LRG_321t8, which means that all eight individual TP53 reference transcripts can be quantitated in cell lines and fresh frozen tumor samples. We use this information to provide new insights into the impact of individual TP53 transcripts and TP53 gene mutations, as well as their interplay, on breast cancer patient outcome.

Given that a recent report described frequent transcription of TP53 DNA mutations into TP53 RNA [28], for most tumors in this study we have assumed that the TP53 DNA mutations will be transcribed into RNA, unless the mutations influence RNA stability. However, for a subset of tumors in this study (the five with TP53 splicing mutations), we confirmed transcription into RNA. Although the proportion of breast tumors with a TP53 mutation is consistent with international data, the proportion of these that are splice site mutations in the NZ cohort is considerably higher [2-4]. We observed that $16 \%$ of the TP53 mutations would influence TP53 transcript splicing, compared to $2 \%$ reported in public databases $[4,19,29]$, and even a recent publication that showed that splice mutations accounted for $6.6 \%$ of TP53 mutations in colorectal cancer [30]. One explanation for this may be that not all mutation callers are reporting mutations that will influence splicing. Other sites located around the splice junctions or within the intron may influence splicing, but are not reported as has been described $[19,30,31]$. In addition, in silico methods used in previous studies may underestimate the effects of mutations on splicing [32].

Our results suggest that TP53 intron 4 splicing mutations have a significant effect on the TP53 transcript profile/composition. Although others have described how truncated FL/ $\triangle 40$ p53 isoforms would arise from these mutations [30,33], our data show that these effectively lead to very high levels of the $t 5-t 7$ transcripts, driven off the TP53 P1 promoter, and therefore predict that very high levels of the $\Delta 133$ p53 isoforms would be expressed. A recent paper has highlighted the clinical relevance of TP53 intron 4 splicing mutations. During platinum/paclitaxel-based neo-adjuvant treatment for high grade serous ovarian cancer, an intron 4 donor splice mutation (c. $375+1 \mathrm{G}>\mathrm{A}$ ) became the highly proliferative major clone, suggesting that this TP53 mutation was potentially associated with chemotherapy resistance [33]. A TP53 mutation influencing the intron 4 donor splice site has also been reported in a Li-Fraumeni family [34].

Using long amplicon ddPCR methods also allowed us to examine how TP53 splice site mutations manifest themselves in the transcripts expressed from the locus. In addition to those TP53 mutations influencing the splicing of large introns, which are clearly visible in long amplicon ddPCR assays (such as retention of the $757 \mathrm{bp}$ intron 4; [13]), another example was sample AL0073 where a splice site mutation in 39\% of the TP53 gene sequencing reads led to $82 \%$ of transcripts retaining intron 7 , despite no evidence of $\mathrm{LoH}$. As exons 5-9 are present in all TP53 reference transcripts, retention of intron 7 would therefore impact all p53 isoforms expressed. For the intron 5 acceptor splice site mutation in sample AL0068, we saw no evidence of intron 5 retention, but instead the results suggested that exon 6 was skipped in a proportion of transcripts. This same mutation has been reported in colorectal cancers, where in the TP53 RNA-seq data, exon 6 skipping was a more frequent event than intron 5 retention [30]. Our assays were sufficiently sensitive to detect intron 5 retention but did not, suggesting that exon 6 skipping is the predominant effect of this mutation.

We also showed that in addition to splice mutations, other TP53 mutations also alter the proportion/composition of the TP53 transcripts in the tumor. By analyzing the endogenous full length TP53 transcripts, our results tentatively suggest frameshift mutations may affect the levels of the FL/ $\Delta 40 \mathrm{p} 53 \alpha$ - and FL/ $\Delta 40 \mathrm{p} 53 \beta$-encoding transcripts differently. Consistent with other studies $[4,7,30]$, the abundance of transcripts encoding p53 isoforms with an $\alpha \mathrm{C}$-terminal end showed that those with a frameshift mutation were present at lower levels than those $\alpha$-encoding transcripts with a missense or no TP53 mutation. The explanation for this has been proposed to be that transcripts with frameshift mutations are subjected to nonsense-mediated decay (NMD) [4,30]. However, we observed that the $t 3$ transcript levels (encoding FL/ $\triangle 40 \mathrm{p} 53 \beta$ ) were similar irrespective of the type of TP53 mutation. These findings suggest that endogenous $t 3$ transcripts with a frameshift 
mutation may be less susceptible to NMD. Therefore, certain TP53 mutation types may not only alter the sequence but also the overall composition of the TP53 isoforms expressed in a tumor. If the $\beta$-encoding TP53 transcripts with frameshift mutations are not subjected to NMD, this is important because recent papers have shown that neo-open reading frames arise from transcripts with frameshift TP53 mutations [35]. Therefore, the $t 3$ transcript may be a template for this phenomenon.

As shown by others [6,36], we observed that TP53 mutational status was associated with prognosis, as patients having tumors with a TP53 mutation were more likely to develop metastases than those with no TP53 mutation. Our analysis of the associations of the individual TP53 transcripts with breast cancer patient prognosis showed some results consistent with other studies reporting data from TP53 $3^{\prime}$ end assays. For example, we observed that patients with a TP53 tumor mutation and less than median (including undetectable) levels of transcripts $t 3$ and $t 4$ (encoding FL/ $\Delta 40 \mathrm{p} 53 \beta$ and FL/ $\Delta 40 \mathrm{p} 53 \gamma$, respectively) had very poor prognosis, consistent with previous findings from analysis of the TP53 $\beta$ and TP53 $\gamma 3^{\prime}$ ends [14,15]. However, in contrast to previous studies $[14,15]$, we did not observe that patients with tumors with high $t 3$ or detectable $t 4$ levels and TP53 mutation had the best disease-free or breast cancer specific survival rates. In addition, detectable levels of the $t 6$ transcript encoding $\Delta 133 p 53 \beta$ by semi-quantitative PCR have been associated with breast cancer patient outcome [16], although analysis of $t 6$ transcript levels in our cohort was not associated with prognosis. Furthermore, we did not observe the association of these transcripts with prognosis in multivariable analysis across the entire cohort with or without interaction terms. This discrepancy (from analysis of patient subsets compared to analysis of all patients) indicates the association is less statistically robust and more likely due to cohort effects. There are several possible explanations for the inconsistencies between these results. Firstly, most studies have used RT-qPCR and we have used ddPCR. Furthermore, these previous studies have predominantly measured the TP53 $3^{\prime}$ ends, which are providing information from multiple transcripts, whereas our assays are measuring individual transcripts. In addition, the NZ population is a distinctly unique group of ethnicities [37]; for TP53 this is apparent given the proportion of TP53 splicing mutations in our cohort being considerably higher than in international cohorts.

Significantly, we found that the ratio of the $t 2 / t 1$ transcripts and TP53 mutation status of breast tumors were associated with distant metastases free survival of patients, independent of any clinical and pathological features. This TP53 information also contributed more strongly than most clinical and pathological features, including tumor grade and ER status, to a model that predicted DMFS. The best multivariable Cox proportional hazards regression model (i.e., the model that explained the highest proportion of variance in patient outcome) was a combination of the ratio of the $t 2 / t 1$ transcripts and TP53 mutation status of the breast tumors with tumor size and patient lymph node status. We also took an alternative approach to show how this TP53 information was associated with DMFS, by stratifying patients into four groups based on their tumors having above or below $t 2 / t 1$ median levels and TP53 mutation or not. This showed two groups that had very different outcomes, those patients with high $t 2 / t 1$ tumor levels and TP53 mutation had frequent metastases, but a group of 28 patients (representing $~ 1 / 3$ of the cohort) with low t2 / $t 1$ tumor levels and no TP53 mutation where only one patient developed metastases. Although studies have suggested that tumor TP53 mutation status is not always a good indicator of prognosis [4,19], our data suggests that incorporating information from certain TP53 transcripts, such as the $t 2 / t 1$ ratio, in addition to TP53 mutation status of the tumor, may be a more accurate predictor of patient prognosis than clinical and pathological information alone.

The main limitation of our study is the small cohort size, therefore any conclusions may not be substantiated in studies of larger breast cancer cohorts. This is especially so in our analyses that stratified the cohort into four subgroups, as discussed above. Therefore, our findings need to be followed up in larger cohorts of breast cancer patient samples, exploiting the long amplicon ddPCR assays to quantitate the individual TP53 transcripts 
in parallel to generating information about TP53 mutation status and copy number, to provide a truly integrative perspective of the biological roles and clinical implications of TP53 isoforms in breast cancer.

\section{Materials and Methods}

\subsection{Patient Samples and Human Ethics}

Breast cancer samples were a subset of our previous study [24], with informed written consent obtained from all study subjects and ethical approval granted by the New Zealand Multi-Region Ethics Committee (project MEC 09/06/060). Patient follow up data was obtained from the Breast Cancer Foundation NZ National Register. Clinical and pathological features of the patient samples used in this study are described in Table S3.

\subsection{TP53 Gene Sequencing}

Genomic DNA was prepared from the breast tumor samples described in [24], using Trizol (Thermo Fisher, Waltham, MA, USA) according to the manufacturer's instructions. For each sample, RNA had previously been isolated from the aqueous phase during the Trizol extraction procedure [13,24], and genomic DNA was then isolated from the remaining organic phase. The concentration of the genomic DNA was determined by Qubit (Thermo Fisher) and the quality of the DNA analyzed by gel electrophoresis. Next generation sequencing of the TP53 gene was performed using a custom Ion AmpliSeq panel (covering TP53 and TP63 genes). Libraries were generated using the Ion AmpliSeq Library Kit 2.0 (Thermo Fisher) according to the manufacturer's specifications, with $50 \mathrm{ng}$ of genomic DNA as input. Two libraries were prepared with 48 samples in each. The libraries were purified using AMPure XP beads (Agencourt Bioscience, Beverly, MA, USA) and then quantified using the IonLibrary TaqMan Quantitation kit (Thermo Fisher). Sequencing was performed on the Ion Torrent Proton machine (Thermo Fisher) running 48 samples on two Ion P1 chips (Thermo Fisher) according to the manufacturer's instructions. Data analysis, including alignment to the hg19 human reference genome and mutation calling, was performed in the Ion Reporter Software v.5.4 (Thermo Fisher). The mutation allele frequency cut-off was set at $>10 \%$, without adjusting for tumor cellularity. All mutations were confirmed manually using the Integrative Genomics Viewer [38].

\subsection{Digital PCR Assays}

RNA was isolated from fresh frozen breast tumors using TRIzol reagent (Thermo Fisher) and cDNA synthesis performed with $1.5 \mu \mathrm{g}$ RNA in $20 \mu \mathrm{L}$ reaction volume using SuperScript IV (Thermo Fisher) both as previously described [13]. Long amplicon ddPCR assays were used to quantitate $L R G_{-} 321 t 1-t 7$ TP53 transcripts in breast tumors (Table S4) as described in [13]. The TP53 $5^{\prime}$ and $3^{\prime}$ end ddPCR assays were performed as previously described [12].

\subsubsection{Long Amplicon ddPCR Methodology}

For each sample, a $22 \mu \mathrm{L}$ reaction mix was prepared consisting of $11 \mu \mathrm{L} 2 \times$ ddPCR Supermix for Probes (no dUTP) (Bio-Rad, Hercules, CA, USA), $1 \mu \mathrm{L} 20 \mu \mathrm{M}$ Forward primer, $1 \mu \mathrm{L} 20 \mu \mathrm{M}$ Reverse primer and $0.28 \mu \mathrm{L}$ each $20 \mu \mathrm{M}$ probe (Table 4 and Table 6 ). DNase/RNase-free water was added to a final volume of $17 \mu \mathrm{L}$. To this, $5 \mu \mathrm{L}$ cDNA diluted in DNase/RNase-free water was added. The droplets were generated and PCR performed as previously described [13], with cycling conditions for each assay described below. Following PCR, the droplets from each well were read on the QX200 droplet reader (Bio-Rad) and data analyzed with QuantaSoft Analysis Pro Software (version 1.0.596) according to manufacturer's instructions. The threshold was set manually to discriminate between negative and positive droplets. cDNA was substituted with RNase-free water as a non-template control for each probe set, to allow for gating of negative droplets for analysis. The data was visualised in 1-D and 2-D plots using the QuantaSoft Analysis Pro 
Software [39]. Data from any wells with $<10,000$ droplets were discarded. Quantitated transcripts were calculated to give the concentration as copies/ $\mu \mathrm{g}$ of RNA.

Table 4. Description of primers and probes for TP53 splice mutation long amplicon ddPCR assays.

\begin{tabular}{|c|c|c|c|}
\hline Assay (Intron) & Oligonucleotide & TP53 Location & Sequence $\left(5^{\prime}-3^{\prime}\right)$ \\
\hline 4 & Forward primer ${ }^{1}$ & Exon 3 & ACTTCCTGAAAACAACGTTCTG \\
\hline 4 & Reverse primer ${ }^{1}$ & Exon 6 & CCACACGCAAATTTCCTTCC \\
\hline 4 & Probe_HEX ${ }^{1}$ & Exon 4 & 5HEX_TGCCCTGGTAGGTTTTCTGGGAAGGGAC_3IABkFQ \\
\hline 5 & Forward primer ${ }^{2}$ & Exon 5 & CAGCTGTGGGTTGATTCCA \\
\hline 5 & Reverse primer ${ }^{2}$ & Exon 7 & GTGATGATGGTGAGGATGGG \\
\hline 5 & Probe_HEX ${ }^{2}$ & Exon 5 & 5HEX_TGCTTGTAGATGGCCATGGC_3IABkFQ \\
\hline 7 & Forward primer ${ }^{3}$ & Exon 7 & CССАТССТСАССАТСАТСАС \\
\hline 7 & Reverse primer ${ }^{3}$ & Exon 8 & GTGAGGCTCСССTTTCTTG \\
\hline 7 & Probe_HEX ${ }^{3}$ & Exon 8 & 5HEX_ATTCTCTTCCTCTGTGCGCC_3IABkFQ \\
\hline
\end{tabular}

For assay to detect TP53 RNAs expressed in tumors with the following splice site mutations; ${ }^{1}$ Intron $4{ }^{2}$ Intron $5,{ }^{3}$ Intron 7 .

4.3.2. Long Amplicon ddPCR Assays to Detect TP53 RNAs Expressed in Tumors with TP53 Gene Splice Site Mutations

Three assays were designed to detect the TP53 transcripts expressed in the tumors that had splice site mutations reported in the TP53 gene sequence. These were splice site mutations that were in intron 4 , intron 5 and intron 7. The ddPCR assays were set up as described above, using $5 \mu \mathrm{L}$ cDNA diluted 1:20 in DNase/RNase-free water, or 2ng genomic DNA as a control. Primers and probes for each assay are shown in Table 4 and ddPCR cycling conditions in Table 5.

Table 5. Long amplicon ddPCR cycling conditions for the TP53 splice mutation assays.

\begin{tabular}{|c|c|}
\hline Assay & ddPCR Cycling Conditions \\
\hline Intron 4 splice mutation & $\begin{array}{l}94^{\circ} \mathrm{C} \text { for } 10 \mathrm{~min}, \\
50 \text { cycles of } 94^{\circ} \mathrm{C} \text { for } 30 \mathrm{~s}, 64^{\circ} \mathrm{C} \text { for } 1 \mathrm{~min}, 72{ }^{\circ} \mathrm{C} \text { for } 6 \mathrm{~min} \text {, } \\
98^{\circ} \mathrm{C} \text { for } 10 \mathrm{~min} \text { and then } 12^{\circ} \mathrm{C} \text { hold }\end{array}$ \\
\hline Intron 5 splice mutation & $\begin{array}{l}94{ }^{\circ} \mathrm{C} \text { for } 10 \mathrm{~min}, \\
50 \text { cycles of } 94^{\circ} \mathrm{C} \text { for } 30 \mathrm{~s}, 62^{\circ} \mathrm{C} \text { for } 30 \mathrm{~s}, 72{ }^{\circ} \mathrm{C} \text { for } 1 \mathrm{~min} \text {, } \\
98^{\circ} \mathrm{C} \text { for } 10 \mathrm{~min} \text { and then } 12^{\circ} \mathrm{C} \text { hold }\end{array}$ \\
\hline Intron 7 splice mutation & $\begin{array}{l}94{ }^{\circ} \mathrm{C} \text { for } 10 \mathrm{~min}, \\
50 \text { cycles of } 94{ }^{\circ} \mathrm{C} \text { for } 30 \mathrm{~s}, 62^{\circ} \mathrm{C} \text { for } 1 \mathrm{~min}, 72{ }^{\circ} \mathrm{C} \text { for } 3 \mathrm{~min} \text {, } \\
98^{\circ} \mathrm{C} \text { for } 10 \mathrm{~min} \text { and then } 12^{\circ} \mathrm{C} \text { hold }\end{array}$ \\
\hline
\end{tabular}

4.3.3. Long Amplicon Multiplex ddPCR Assay to Quantitate the TP53 t8 Transcripts

A long amplicon ddPCR assay was designed to quantitate the TP53 LRG_321t8 transcript (NCBI transcript identifier NM_001126118.1) encoding $\Delta 40 p 53 \alpha$, and also any $\beta$ or $\gamma$ versions of this (called $t 8 \beta$ and $t 8 \gamma$, respectively). The ddPCR assay was set up as described above, using $5 \mu \mathrm{L}$ cDNA diluted 1:10 in DNase/RNase-free water. The assay primers and probes sequences are shown in Table 6.

Table 6. Description of primers and probes for TP53 $t 8, t 8 \beta$ and $t 8 \gamma$ long amplicon ddPCR assay.

\begin{tabular}{|c|c|c|}
\hline Oligonucleotide & Location & Sequence $\left(5^{\prime}-3^{\prime}\right)$ \\
\hline Forward primer & Intron 2 & AGTGGATCCATTGGAAGGGCAGGC \\
\hline Reverse primer ${ }^{1}$ & Exon 10 & CTGGGCATCCTTGAGTTCC \\
\hline$\alpha$ probe_HEX ${ }^{1}$ & Exons 9/10 & 5HEX_CGGATCTGAAGGGTGAAATATTCTCCA_3IABkFQ \\
\hline$\beta$ probe_FAM_1 1 & Exon $9 \beta$ & 56-FAM_ACTTTGCCTGATACAGATGCTACT_3IABkFQ \\
\hline$\beta$ probe_FAM_2 1 & Exon $9 \beta$ & 56-FAM_TCTGTATCAGGCAAAGTCATAGAACCAT_3IABkFQ \\
\hline$\gamma$ probe_FAM ${ }^{1}$ & Exons $9 / 9 \gamma$ & 56-FAM_AGCATCTGAAGGGTGAAATATTCTCCA_3IABkFQ \\
\hline
\end{tabular}

\footnotetext{
${ }^{1}$ Reverse primer and probes the same as $T P 53 t 1 / t 3 / t 4$ and $t 5 / t 6 / t 7$ multiplex long amplicon ddPCR assays [13].
} 
ddPCR cycling conditions were $94{ }^{\circ} \mathrm{C}$ for $10 \mathrm{~min}$, then 50 cycles of $94{ }^{\circ} \mathrm{C}$ for $30 \mathrm{~s}, 64^{\circ} \mathrm{C}$ for $1 \mathrm{~min}, 72^{\circ} \mathrm{C}$ for $6 \mathrm{~min}$, followed by $98^{\circ} \mathrm{C}$ for $10 \mathrm{~min}$ and then hold temperature of $12^{\circ} \mathrm{C}$. The data was quantitated from analysis of 2-D plots (Table S4). As two probes were run in the FAM channel to allow concurrent quantitation of $t 8 \beta$ and $t 8 \gamma$ transcripts, analysis was performed using the Advanced Classification Method in the QuantaSoft Analysis Pro Software [39].

\subsection{Bioinformatics and Statistical Analysis}

TCGA BRCA gene expression level 3 data was downloaded on 06/10/2019 [20,21] with TP53 mutation information downloaded from UCSC Xena [40] on 23042020. Data was plotted using either R or GraphPad Prism8. Nominal variable association of TP53 transcript information with standard clinical and pathological features was performed using either ANOVA (for breast cancer subtype and histological grade), $t$-test (for tumor ER or PgR status, or patient LN status) or correlation analysis (for patient age at diagnosis). The association of tumor TP53 information (TP53 mutation status, the individual TP53 transcripts or the TP53 $5^{\prime}$ or $3^{\prime}$ ends) with the time to a distant metastatic event was determined using Cox proportional hazards regression models [41,42]. Note that samples with any missing clinical, pathological or TP53 information $(n=4)$ were omitted from this analysis. TP53 information that was a significant prognostic factor in univariable analysis was included with the potential clinical and pathological confounders in a multivariable Cox proportional hazards regression model. All statistical tests were 2-sided, and significance was defined as an alpha of 0.05. Analyses were performed in the R environment [43].

\section{Conclusions}

We have performed an integrated analysis of the TP53 locus in a New Zealand breast cancer cohort, by sequencing both the TP53 gene and quantitating the individual TP53 transcripts expressed from the locus, and combining this with patient clinical and pathological information. Novel multiplex long amplicon ddPCR assay were devised to detect the eighth TP53 reference transcript, $L R G \_321 t 8$, encoding the $\Delta 40 \mathrm{p} 53 \alpha$ isoform, and also transcripts encoding $\beta$ and $\gamma$ versions of this isoform. In the cohort analyzed here, we observed that $16 \%$ of TP53 mutations affect splicing to generate additional TP53 transcripts, as confirmed in long amplicon ddPCR assays. Those mutations that influenced TP53 intron 4 splicing were associated with overexpression of transcripts encoding the $\Delta 133 p 53$ isoforms. We show that incorporating TP53 transcript information in addition to TP53 mutation status may be a more accurate predictor of breast cancer patient prognosis.

Supplementary Materials: The following are available online at https: / www.mdpi.com/2072-6 694/13/7/1531/s1, Figure S1: Analysis of transcripts encoding $\Delta 40$ p53 isoforms, Figure S2: TP53 transcript levels by breast cancer PAM50 subtype, Figure S3: Association of TP53 mutation status and TP53 $t 3$ or $t 4$ transcript levels with breast cancer patient prognosis, Table S1: Description of TP53 gene mutations and loss of the wild type TP53 allele in New Zealand breast tumor cohort, Table S2: Association of TP53 tumor information with clinical, pathological and intrinsic subtype information in New Zealand breast tumor cohort, Table S3: Clinical and pathological features of 89 breast cancer patients in this study, Table S4: Results from long amplicon ddPCR assays to quantitate 10 TP53 transcripts in breast cancer samples.

Author Contributions: Conceptualization, A.L., S.Y.M., A.W.B. and C.G.P.; methodology, A.L. and S.Y.M.; validation, A.L. and N.K.; formal analysis, A.L., N.K. and C.G.P.; investigation, A.L.; resources, A.L. and S.Y.M.; data curation, A.L. and N.K.; writing—original draft preparation, A.L.; writingreview and editing, A.L., N.K., S.Y.M., A.W.B. and C.G.P.; visualization, A.L.; project administration, A.L. and C.G.P.; funding acquisition, A.W.B. and C.G.P. All authors have read and agreed to the published version of the manuscript.

Funding: This work was funded by the Health Research Council of New Zealand Programme Grant $15 / 500$. 
Institutional Review Board Statement: This study was conducted according to the guidelines of the Declaration of Helsinki, and approved by the New Zealand Multi-Region Ethics Committee (project MEC 09/06/060, approved 2009).

Informed Consent Statement: Informed consent was obtained from all subjects involved in the study.

Data Availability Statement: The data presented in this study are available in Table S1, Table S3, Table S4 and [24].

Acknowledgments: We thank the Breast Cancer Foundation National Register for provision of the data, and the Breast Cancer Foundation NZ as the funder of the Breast Cancer Foundation National Register. We also thank the Breast Cancer Cure NZ for their long term support of this research program, which made this paper possible.

Conflicts of Interest: The authors declare no conflict of interest. The funders had no role in the design of the study; in the collection, analyses, or interpretation of data; in the writing of the manuscript, or in the decision to publish the results.

\section{References}

1. Kandoth, C.; McLellan, M.D.; Vandin, F.; Ye, K.; Niu, B.; Lu, C.; Xie, M.; Zhang, Q.; McMichael, J.F.; Wyczalkowski, M.A.; et al. Mutational landscape and significance across 12 major cancer types. Nature 2013, 502, 333-339. [CrossRef]

2. Bouaoun, L.; Sonkin, D.; Ardin, M.; Hollstein, M.; Byrnes, G.; Zavadil, J.; Olivier, M. TP53Variations in Human Cancers: New Lessons from the IARC TP53 Database and Genomics Data. Hum. Mutat. 2016, 37, 865-876. [CrossRef]

3. Pereira, B.; Chin, S.-F.; Rueda, O.M.; Vollan, H.-K.M.; Provenzano, E.; Bardwell, H.A.; Pugh, M.; Jones, L.A.; Russell, R.; Sammut, S.-J.; et al. The somatic mutation profiles of 2433 breast cancers refine their genomic and transcriptomic landscapes. Nat. Commun. 2016, 7, 11479. [CrossRef]

4. Donehower, L.A.; Soussi, T.; Korkut, A.; Liu, Y.; Schultz, A.; Cardenas, M.; Li, X.; Babur, O.; Hsu, T.K.; Lichtarge, O.; et al. Integrated Analysis of TP53 Gene and Pathway Alterations in The Cancer Genome Atlas. Cell Rep. 2019, 28, 1370-1384. [CrossRef]

5. Banerji, S.; Cibulskis, K.; Rangel-Escareno, C.; Brown, K.K.; Carter, S.L.; Frederick, A.M.; Lawrence, M.S.; Sivachenko, A.Y.; Sougnez, C.; Zou, L.; et al. Sequence analysis of mutations and translocations across breast cancer subtypes. Nature 2012, 486, 405-409. [CrossRef]

6. $\quad$ Silwal-Pandit, L.; Vollan, H.K.M.; Chin, S.-F.; Rueda, O.M.; McKinney, S.; Osako, T.; Quigley, D.A.; Kristensen, V.N.; Aparicio, S.; Børresen-Dale, A.-L.; et al. TP53 Mutation Spectrum in Breast Cancer Is Subtype Specific and Has Distinct Prognostic Relevance. Clin. Cancer Res. 2014, 20, 3569-3580. [CrossRef] [PubMed]

7. Mehta, S.Y.; Morten, B.C.; Antony, J.; Henderson, L.; Lasham, A.; Campbell, H.; Cunliffe, H.; Horsfield, J.A.; Reddel, R.R.; Avery-Kiejda, K.A.; et al. Regulation of the interferon-gamma (IFN-gamma) pathway by p63 and Delta133p53 isoform in different breast cancer subtypes. Oncotarget 2018, 9, 29146-29161. [CrossRef] [PubMed]

8. Joruiz, S.M.; Bourdon, J.-C. p53 Isoforms: Key Regulators of the Cell Fate Decision. Cold Spring Harb. Perspect. Med. 2016, 6, a026039. [CrossRef] [PubMed]

9. Zerbino, D.R.; Achuthan, P.; Akanni, W.; Amode, M.R.; Barrell, D.; Bhai, J.; Billis, K.; Cummins, C.; Gall, A.; Girón, C.G.; et al. Ensembl 2018. Nucleic Acids Res. 2018, 46, D754-D761. [CrossRef] [PubMed]

10. Dalgleish, R.; Flicek, P.; Cunningham, F.; Astashyn, A.; Tully, R.E.; Proctor, G.; Chen, Y.; McLaren, W.M.; Larsson, P.; Vaughan, B.W.; et al. Locus Reference Genomic sequences: An improved basis for describing human DNA variants. Genome Med. 2010, 2, 24-27. [CrossRef]

11. Anbarasan, T.; Bourdon, J.-C. The Emerging Landscape of p53 Isoforms in Physiology, Cancer and Degenerative Diseases. Int. J. Mol. Sci. 2019, 20, 6257. [CrossRef]

12. Mehta, S.; Tsai, P.; Lasham, A.; Campbell, H.; Reddel, R.; Braithwaite, A.; Print, C. A Study of TP53 RNA Splicing Illustrates Pitfalls of RNA-seq Methodology. Cancer Res. 2016, 76, 7151-7159. [CrossRef] [PubMed]

13. Lasham, A.; Tsai, P.; Fitzgerald, S.J.; Mehta, S.Y.; Knowlton, N.S.; Braithwaite, A.W.; Print, C.G. Accessing a New Dimension in TP53 Biology: Multiplex Long Amplicon Digital PCR to Specifically Detect and Quantitate Individual TP53 Transcripts. Cancers 2020, 12, 769. [CrossRef]

14. Avery-Kiejda, K.A.; Morten, B.; Wong-Brown, M.W.; Mathe, A.; Scott, R.J. The relative mRNA expression of p53 isoforms in breast cancer is associated with clinical features and outcome. Carcinogenesis 2013, 35, 586-596. [CrossRef]

15. Bourdon, J.-C.; Khoury, M.P.; Diot, A.; Baker, L.; Fernandes, K.; Aoubala, M.; Quinlan, P.; Purdie, A.C.; Jordan, L.B.; Prats, A.-C.; et al. p53 mutant breast cancer patients expressing p53 $\gamma$ have as good a prognosis as wild-type p53 breast cancer patients. Breast Cancer Res. 2011, 13, R7. [CrossRef]

16. Gadea, G.; Arsic, N.; Fernandes, K.; Diot, A.; Joruiz, S.M.; Abdallah, S.; Meuray, V.; Vinot, S.; Anguille, C.; Remenyi, J. Tp53 drives invasion through expression of its $\Delta 133$ p53 $\beta$ variant. eLife 2016, 5, e14734. [CrossRef] [PubMed]

17. Razavi, P.; Chang, M.T.; Xu, G.; Bandlamudi, C.; Ross, D.S.; Vasan, N.; Cai, Y.; Bielski, C.M.; Donoghue, M.T.; Jonsson, P.; et al. The Genomic Landscape of Endocrine-Resistant Advanced Breast Cancers. Cancer Cell 2018, 34, 427-438.e6. [CrossRef] [PubMed] 
18. Curtis, C.; Shah, S.P.; Chin, S.F.; Turashvili, G.; Rueda, O.M.; Dunning, M.J.; Speed, D.; Lynch, A.G.; Samarajiwa, S.; Yuan, Y.; et al. The genomic and transcriptomic architecture of 2000 breast tumours reveals novel subgroups. Natature 2012, 486, 346-352. [CrossRef]

19. Leroy, B.; Anderson, M.; Soussi, T. TP53 Mutations in Human Cancer: Database Reassessment and Prospects for the Next Decade. Hum. Mutat. 2014, 35, 672-688. [CrossRef]

20. Grossman, R.L.; Heath, A.P.; Ferretti, V.; Varmus, H.E.; Lowy, D.R.; Kibbe, W.A.; Staudt, L.M. Toward a Shared Vision for Cancer Genomic Data. N. Engl. J. Med. 2016, 375, 1109-1112. [CrossRef]

21. TCGA BRCA Gene Expression Level 3 Data. Available online: https:/ / gdac.broadinstitute.org/ (accessed on 10 June 2019).

22. Ghosh, A.; Stewart, D.; Matlashewski, G. Regulation of Human p53 Activity and Cell Localization by Alternative Splicing. Mol. Cell. Biol. 2004, 24, 7987-7997. [CrossRef] [PubMed]

23. Sorlie, T.; Tibshirani, R.; Parker, J.; Hastie, T.; Marron, J.S.; Nobel, A.; Deng, S.; Johnsen, H.; Pesich, R.; Geisler, S.; et al. Repeated observation of breast tumor subtypes in independent gene expression data sets. Proc. Natl. Acad. Sci. USA 2003, 100, 8418-8423. [CrossRef] [PubMed]

24. Muthukaruppan, A.; Lasham, A.; Blenkiron, C.; Woad, K.J.; Black, M.A.; Knowlton, N.; McCarthy, N.; Findlay, M.P. Genomic profiling of breast tumours from New Zealand patients. N. Z. Med. J. 2017, 130, 40-56.

25. Haybittle, J.L.; Blamey, R.W.; Elston, C.W.; Johnson, E.J.; Doyle, P.J.; Campbell, F.C.; Nicholson, I.R.; Griffiths, K. A prognostic index in primary breast cancer. Br. J. Cancer 1982, 45, 361-366. [CrossRef]

26. Ravdin, P.M.; Siminoff, L.A.; Davis, G.J.; Mercer, M.B.; Hewlett, J.; Gerson, N.; Parker, H.L. Computer Program to Assist in Making Decisions About Adjuvant Therapy for Women with Early Breast Cancer. J. Clin. Oncol. 2001, 19, 980-991. [CrossRef]

27. Wishart, G.C.; Azzato, E.M.; Greenberg, D.C.; Rashbass, J.; Kearins, O.; Lawrence, G.; Caldas, C.; Pharoah, P.D. Predict: A new UK prognostic model that predicts survival following surgery for invasive breast cancer. Breast Cancer Res. BCR 2010, 12, 1-10. [CrossRef]

28. Calabrese, C.; Davidson, N.R.; Demircioglu, D.; Fonseca, N.A.; He, Y.; Kahles, A.; Lehmann, K.V.; Liu, F.; Shiraishi, Y.; Soulette, C.M.; et al. Genomic basis for RNA alterations in cancer. Nat. Cell Biol. 2020, 578, 129-136. [CrossRef]

29. Olivier, M.; Bouaoun, L.; Sonkin, D.; Ardin, M.; Hollstein, M.; Byrnes, G.; Zavadil, J. TP53 variations in human cancers: New lessons from the IARC TP53 Database and genomic studies. Eur. J. Cancer 2016, 61, S15. [CrossRef]

30. Smeby, J.; Sveen, A.; Eilertsen, I.A.; Danielsen, S.A.; Hoff, A.M.; Eide, P.W.; Johannessen, B.; Hektoen, M.; Skotheim, R.I.; Guren, M.G.; et al. Transcriptional and functional consequences of TP53 splice mutations in colorectal cancer. Oncogenesis 2019, 8, 35. [CrossRef]

31. Jayasinghe, R.G.; Cao, S.; Gao, Q.; Wendl, M.C.; Vo, N.S.; Reynolds, S.M.; Zhao, Y.; Climente-González, H.; Chai, S.; Wang, F.; et al. Systematic Analysis of Splice-Site-Creating Mutations in Cancer. Cell Rep. 2018, 23, 270-281.e3. [CrossRef]

32. Soukarieh, O.; Gaildrat, P.; Hamiet, M.; Drouet, A.; Baert-Desurmont, S.; Frébourg, T.; Tosi, M.; Martins, A. Exonic splicing mutations are more prevalent than currently estimated and can be predicted using in silico tools. PLoS Genet. 2016, 12, e1005756. [CrossRef]

33. Garziera, M.; Cecchin, E.; Giorda, G.; Sorio, R.; Scalone, S.; De Mattia, E.; Roncato, R.; Gagno, S.; Poletto, E.; Romanato, L.; et al. Clonal Evolution of TP53 c.375 + 1G > A Mutation in Pre-and Post-Neo-Adjuvant Chemotherapy (NACT) Tumor Samples in High-Grade Serous Ovarian Cancer (HGSOC). Cells 2019, 8, 1186. [CrossRef] [PubMed]

34. Frebourg, T.; Barbier, N.; Yan, Y.X.; Garber, E.J.; Dreyfus, M.; Fraumeni, J.; Li, F.P.; Friend, S.H. Germ-line p53 mutations in 15 families with Li-Fraumeni syndrome. Am. J. Hum. Genet. 1995, 56, 608-615. [PubMed]

35. Koster, J.; Plasterk, R.H.A. A library of Neo Open Reading Frame peptides (NOPs) as a sustainable resource of common neoantigens in up to $50 \%$ of cancer patients. Sci. Rep. 2019, 9, 6577. [CrossRef]

36. Duffy, M.J.; Synnott, N.C.; Crown, J. Mutant p53 in breast cancer: Potential as a therapeutic target and biomarker. Breast Cancer Res. Treat. 2018, 170, 213-219. [CrossRef] [PubMed]

37. Statistics New Zealand. Ethnic Group Summaries Reveal New Zealand's Multicultural Make-Up. Available online: https: / / www.stats.govt.nz/news/ethnic-group-summaries-reveal-new-zealands-multicultural-make-up (accessed on 26 January 2021).

38. Thorvaldsdóttir, H.; Robinson, J.T.; Mesirov, J.P. Integrative Genomics Viewer (IGV): High-performance genomics data visualization and exploration. Brief. Bioinform. 2012, 14, 178-192. [CrossRef]

39. Droplet Digital PCR. Laboratories. B.-R. Applications Guide. Available online: https://www.bio-rad.com/webroot/web/pdf/ 1sr/literature/Bulletin_6407.pdf (accessed on 20 March 2018).

40. Goldman, M.J.; Craft, B.; Hastie, M.; Repečka, K.; McDade, F.; Kamath, A.; Banerjee, A.; Luo, Y.; Rogers, D.; Brooks, A.N.; et al. Visualizing and interpreting cancer genomics data via the Xena platform. Nat. Biotechnol. 2020, 38, 675-678. [CrossRef]

41. Harrington, D.; Fleming, T. A class of rank test procedures for censored survival data. Biometrika 1982, 69, 553-566. [CrossRef]

42. Therneau, T. Survival: Survival Analysis, Including Penalized Likelihood; R Package (Version 2.36-5); R foundation for Statistical Computing: Vienna, Austria, 2011.

43. R Core Team. R: A Language and Environment for Statistical Computing; R Foundation for Statistical Computing: Vienna, Austria, 2014. 